\title{
Dust interferometers in plasmas
}

\author{
M. Chaudhuri ${ }^{1 *}$, V. Nosenko ${ }^{2}$, H. M. Thomas ${ }^{2}$ \\ ${ }^{1}$ School of Engineering and Applied Sciences (SEAS), Harvard University, USA and \\ ${ }^{2}$ Forschungsgruppe Komplexe Plasmen, Deutsches Zentrum für Luft- und Raumfahrt, D-82234, Weßling, Germany
}

\begin{abstract}
An interferometric imaging technique has been proposed to measure diameter of individual spherical dust particle instantly inside the plasma chamber. The technique is based on the defocus image analysis of both spherical particles and their binary agglomerates (binary agglomerates). Above a critical diameter, the defocus images of spherical particles contain stationary interference fringe patterns and the fringe number increases with particle diameters. Below this critical diameter, the particle size has been measured using the rotational interference fringe patterns which appears only on defocus images of binary agglomerates. In this case a lower cut-off limit of particle diameter has been predicted below which no such rotational fringe patterns are observed for their binary agglomerates. The method can be useful as a diagnostics for complex plasma experiments on earth as well as under microgravity condition.
\end{abstract}

PACS numbers: 52.27.Lw, 61.20.Ja, 64.70.Dv

Understanding strongly correlated phenomena such as 7 crystal and liquid structures, melting dynamics, crystals lization, homogeneous nucleation, dendrites, glass tran9 sition, etc. in a classical many body systems are out10 standing topics of practical importance in material sci${ }_{11}$ ence [1-4]. Colloids have long been used as a model 12 system to investigate such processes where particles of 13 different shapes (sphere, cube, ellipsoid, agglomerates, 14 etc.) can be synthesized based on experimental require15 ments [5-11]. The surfaces of colloidal particles can be 16 treated chemically to explore wide range of inter-particle ${ }_{17}$ interactions (repulsive to attractive, hard sphere to ultra18 soft) and associated tunable, collective, self-organized 19 processes. Different high resolution imaging techniques, 20 such as laser scanning confocal microscope (LSCM), elec${ }_{21}$ tron microscopy or atomic force microscopy (AFM) tech22 niques can be used to measure size and shape of colloidal 23 particles precisely. Other technique such as dynamic light 24 scattering has also been used to measure hydrodynamic 25 size of the particles in a dilute solution. In recent times, 26 it is revealed that colloids share unique complementary 27 features with complex (dusty) plasma which is being con${ }_{28}$ sidered as the plasma state of soft matter [12-14]. How${ }_{29}$ ever, unlike colloids where the particle dynamics is over so damped due to viscous solvents, the highly charged solid ${ }_{31}$ particles in complex plasmas levitate in the background 32 of weakly ionized gas [15-17]. Basic understanding of 33 plasma-particle interactions are essential to tune inter${ }_{34}$ particle interactions and relevant self-organized collective 35 phenomena in complex plasmas [18-24]. The background 36 neutral gas pressure can be controlled precisely to achieve ${ }_{37}$ almost undamped particle dynamics which makes com38 plex plasma a unique model system to explore classical 39 many body phenomena at the "atomistic" level [14, 25]. 40 Different types of unique experiments have been per${ }_{41}$ formed at ground based laboratories on earth [26-36], 42 as well as under microgravity condition onboard "In43 ternational Space Station (ISS)" [37-41]. Typically ex${ }_{44}$ perimental data in complex plasmas is analyzed by us45 ing standard particle location and tracking methods on
${ }_{46}$ sequence of images obtained by video microscopy tech47 nique. Several other techniques with different features ${ }_{48}$ have also been used, such as, Particle-Image-Velocimetry 49 (PIV) [42, 43], digital in-line holography [44], color gra50 dient method [45], stereoscopy [46], etc. However to the 51 best of our knowledge, there exists no technique in com${ }_{52}$ plex plasmas to identify individual particles shape and 53 size instantly during experiments.

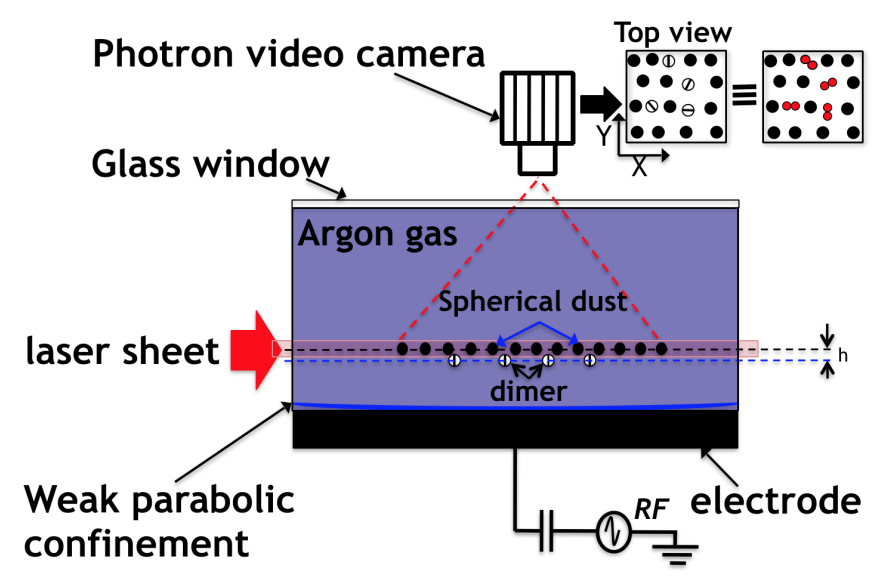

FIG. 1. Sketch of the experimental conditions to prepare quasi-two-dimensional suspension of spherical dust particles and binary agglomerates (binary agglomerates) in the background of weakly ionized plasmas. The microparticles are trapped in the weak parabolic confinement potential above the rf electrode and are illuminated with a horizontal laser sheet. Unlike spherical particles, the binary agglomerates are identified with rotational interference fringe pattern on their defocus images (marked as striped particle on top view image). The spherical particles and binary agglomerates levitate at different heights with separation ' $h$ ' as shown.

${ }_{54}$ Recently defocus imaging technique has been used 55 as an useful diagnostic to identify binary agglomerates 56 in complex plasma which contains rotating interference ${ }_{57}$ fringe patterns on their defocus images [47]. Now, it ${ }_{58}$ is discovered that stationary interference fringes appear 
59 on individual, bigger size, spherical particles. In such ${ }_{60}$ cases, a combination of rotational and stationary fringe 61 patterns are the characteristic of binary agglomerates as 62 shown in Fig. 2f. At some point, the rotational fringe 63 pattern overlaps exactly on top of stationary fringe pat${ }_{64}$ terns which implies that inter-fringe spacings are iden65 tical on defocus images for such spherical particles and ${ }_{66}$ their binary agglomerates. The goal of this work is to 67 put forward an idea of using defocus imaging technique 68 to measure diameter of individual spherical dust parti69 cle instantly during experiments within some accuracy. ${ }_{70}$ We have tried to explore the origin of the fringe patterns 71 for individual spherical dust particle which itself acts as 72 an efficient interferometer. The fringe pattern becomes 73 distinct as the particle diameter increases and there is a 74 lower cut-off $(\sim 9 \mu \mathrm{m})$ below which we don't observe any 75 such fringes. For medium size particles, rotational fringes 76 appear on binary agglomerates but very faint fringes ap77 pear on defocus images of individual spherical particles. 78 For smaller particles (below $\sim 5.5 \mu \mathrm{m}$ ) no fringes are 79 observed at all on either spherical particles or on their 80 binary agglomerates.

${ }_{81}$ The experiments were performed with a (modified) ${ }_{82}$ Gaseous Electronics Conference (GEC) chamber, in a ca${ }_{83}$ pacitively coupled rf glow discharge at $13.56 \mathrm{MHz}$ (see ${ }_{84}$ Fig. 1). The Argon pressure and the forward rf power ${ }_{85}$ were kept at $1 \mathrm{~Pa}$ and at 20 Watt respectively. Parti${ }_{86}$ cles of different sizes and materials have been used for 87 the experiments: Melamine formaldehyde (MF) parti88 cles (mass density: $1.51 \mathrm{gm} / \mathrm{cm}^{3}$, RI: 1.68 ) with diame89 ters $(2 r)$ of $7.16 \mu \mathrm{m}, 8.42 \mu \mathrm{m}, 9.19 \mu \mathrm{m}$ and $14.91 \pm 0.26$ 90 $\mu \mathrm{m}$; polystyrene (PS) particles (mass density: $\sim 1.05$ ${ }_{91} \mathrm{gm} / \mathrm{cm}^{3}$, RI: 1.58$)$ with a diameter of $11.35 \mu \mathrm{m}$ and 92 PMMA particles (mass density: $\sim 1.19 \mathrm{gm} / \mathrm{cm}^{3}$, RI: $\left.{ }_{93} 1.49\right)$ with diameters of $17.02 \pm 0.03 \mu \mathrm{m}$ and $20 \mu \mathrm{m}$. The ${ }_{94}$ particle suspension was illuminated with a horizontal 95 sheet of red diode laser light (wavelength of $660 \mathrm{~nm}$ ) and 96 imaged through the top glass window with a Nikon AF${ }_{97}$ 95S VR Micro-Nikkor camera operating at a speed of 60 ${ }_{98}$ frames/sec with a field of view of $1024 \times 1024$ pixels. The 99 focal length of the lens is $105 \mathrm{~mm}$ with aperture range, $100 f / 2.8$ to $f / 32$. The camera lens was equipped with a 101 narrow-band interference filter to collect only the illumi102 nation laser light scattered by the particles.

${ }_{103}$ When injected in the plasma, both the spherical dust ${ }_{104}$ particles and their binary agglomerates become highly 105 charged and forms a quasi-two dimensional suspension 106 above the lower electrode [47]. The binary agglomerates ${ }_{107}$ levitate just below the monolayer of spherical particles 108 without forming vertical pairs so that all the particles 109 can be viewed from top view camera as shown schemat110 ically in Fig. 1. All the particles can be identified by 111 few bright pixels in a focused image due to laser light 112 scattering. It is not possible to characterize the parti113 cle shape and size by looking at these focused images. ${ }_{114}$ But as we defocus the images, interesting new features 115 are observed: distinct interference fringe patterns appear 116 on the defocused images of the particles [47]. Identical
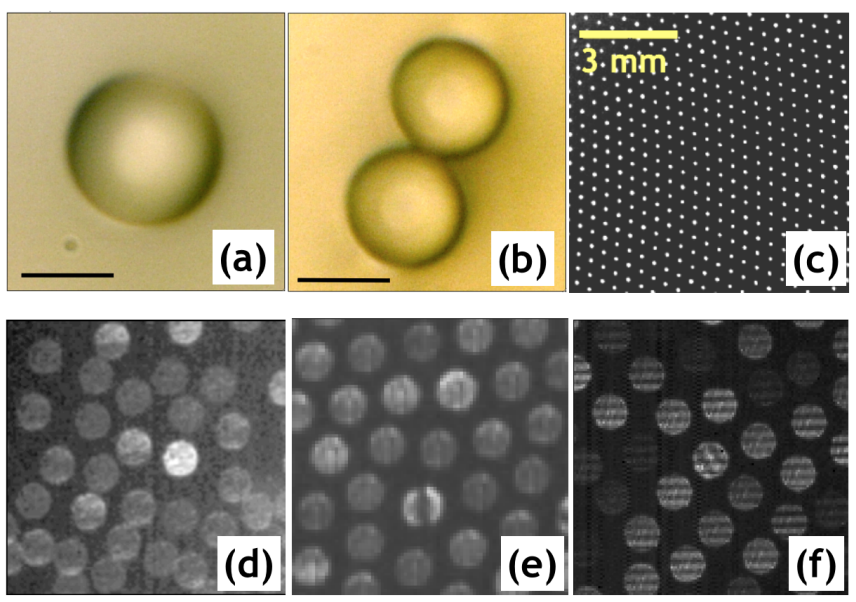

FIG. 2. (a) Spherical dust particle and (b) binary agglomerate as observed through optical microscope. Scale bar is $5 \mu \mathrm{m}$. (c) Two dimensional plasma crystal made of spherical particles as observed in a focused image using video microscopy. (d) Defocused image of small particle where no fringe patterns are observed neither on spherical particles nor on binary agglomerates. (e) Defocused images of medium size particles where rotational fringe patterns are observed on binary agglomerates but not on spherical particles. (f) Large particles where stationary fringe patterns are observed on spherical particles and a combination of rotational fringe on top of stationary fringe patterns are observed on their binary agglomerates.

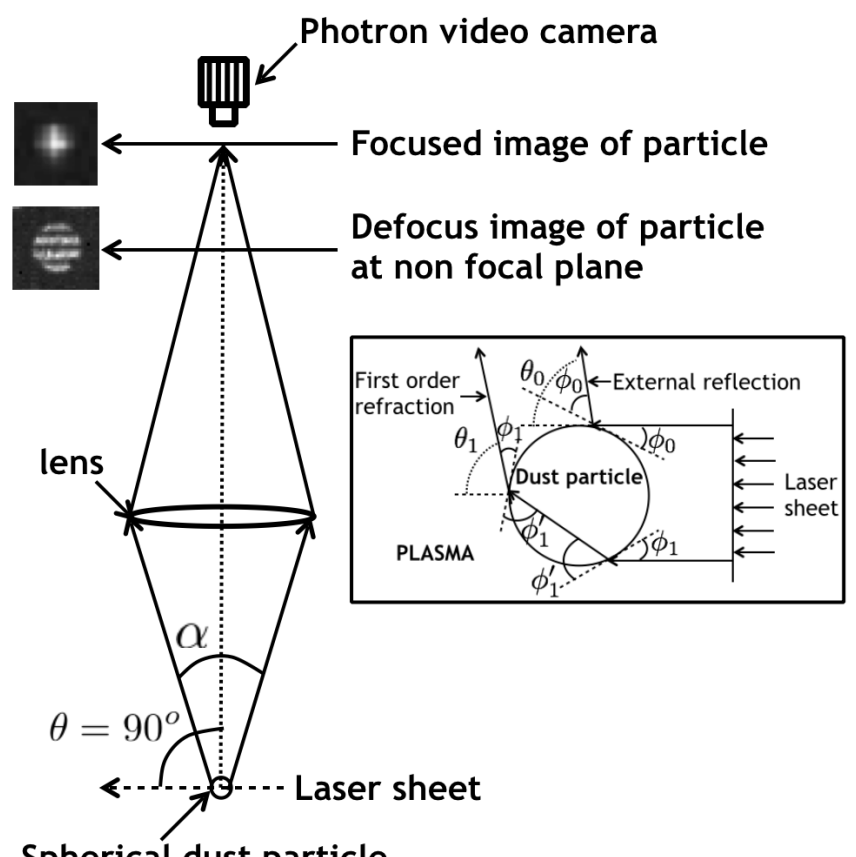

Spherical dust particle

FIG. 3. (a) Schematic diagram of the observed phenomena. The dust particle is illuminated by the laser light and its focussed and defocused images have been visualized by a video camera placed perpendicular to the laser beam. The fringe pattern appears on defocused images due to the interference of reflected and first order refracted light at $90^{\circ}$ scattering angle. Schematics of ray diagram of reflected and first order refracted lights within the dust particle are shown in the inset. 
117 fringe patterns are observed for particles with same di118 ameter as shown in Fig. 2f. As we increase the particle 119 size, the number of fringes also increases on the defocused 120 image of a single spherical particle and they become dis121 tinct. The observed phenomena i.e. the appearance of 122 stationary fringe pattern on bigger size, spherical dust 123 particles in plasma environment has been explained in 124 the framework of "Interferometric Laser Imaging (ILI)" 125 technique which is based on "Mie scattering theory" and 126 takes into account the interference of the scattered light ${ }_{127}$ from a single transparent particle. The reflected and first ${ }_{128}$ order refracted rays interfere with each other to generate 129 fringe patterns at the defocus plane. This technique has 130 been applied before for measuring size of drops and bub131 bles (Interferometric Laser Imaging for Droplet Sizing ${ }_{132}$ (ILIDS)) in spray dryer systems, spark ignition engine, 133 etc. as mentioned in Ref. [48] and references there in. ${ }_{134}$ Two glare points due to reflection and refraction from 135 diametrically opposite positions can be observed at the ${ }_{136}$ focal plane if $d>50 \mu \mathrm{m}$. However, for $d<50 \mu \mathrm{m}$, ILI is ${ }_{137}$ the most suitable technique to determine particle size. It 138 is to be noted that there is a lower limit of particle diam139 eter below which ILI is invalid: $d_{\min } \sim 20 \lambda / \pi$ where $\lambda$ is 140 the wavelength of illumination laser. In our experiment, ${ }_{142}$ we use $\lambda \sim 660 \mathrm{~nm}$ and hence $d_{\min } \sim 4.20 \mu \mathrm{m}$. To cal${ }_{143}$ culate the number of fringes observed on the defocused 144 image of a single particle, we select a one pixel width hor145 izontal region of interest (ROI) along the diameter at the ${ }_{146}$ centre of the particle image. The dark fringes are per147 pendicular to the ROI. The intensity variation along the ${ }_{148}$ ROI exhibits several maxima and minima. As the par149 ticle size increases, the number of fringes increases and 150 hence number of maxima/minima increases: $\mathrm{N} \sim 1.16$ for ${ }_{151} 7.16 \mu \mathrm{m}, \mathrm{N} \sim 1.58$ for $9.19 \mu \mathrm{m}, \mathrm{N} \sim 2.07$ for $11.35 \mu \mathrm{m}$, ${ }_{152} \mathrm{~N} \sim 2.28$ for $14.91 \mu \mathrm{m}, \mathrm{N} \sim 2.88$ for $17.02 \mu \mathrm{m}$, and $\mathrm{N}$ $153 \sim 3.43$ for $20 \mu \mathrm{m}$. It is to be noted that with increasing ${ }_{154}$ particle size, the width of each peak decreases and height 155 increases indicating distinct as well as sharp features of 156 fringe patterns. It is difficult to measure the fringe sep157 aration for smaller particles due to the wider width of ${ }_{158}$ fringes and they appear almost at two ends of ROI.

159 According to Lorentz-Mie theory, the light scattered 160 by a spherical particle is inhomogeneously distributed 161 in space (oscillating function of the angle in the range ${ }_{162} 0<\theta<\pi$ ) which depends on particle diameter, refrac163 tive index and incident light characteristics [49]. The 164 origin of these oscillations is due to interference between 165 reflected, refracted and diffracted rays coming out of the 166 particle and forms the basis of the Mie Scattering Inter167 ferrometry. However, for bigger particle it was shown 168 that simpler geometric analysis can be used as an al169 ternate of complex Mie theory to estimate particle size 170 for a scattering angle centred around $90^{\circ}$. To analyze 171 the phenomenon, we consider all dust particles are per172 fectly spherical and homogeneous. The interaction be173 tween laser beam and the particle is shown in Fig. 3. ${ }_{174}$ The total scattering light intensity is due to the sum of 175 reflection and first order refraction rays. The phase dif-

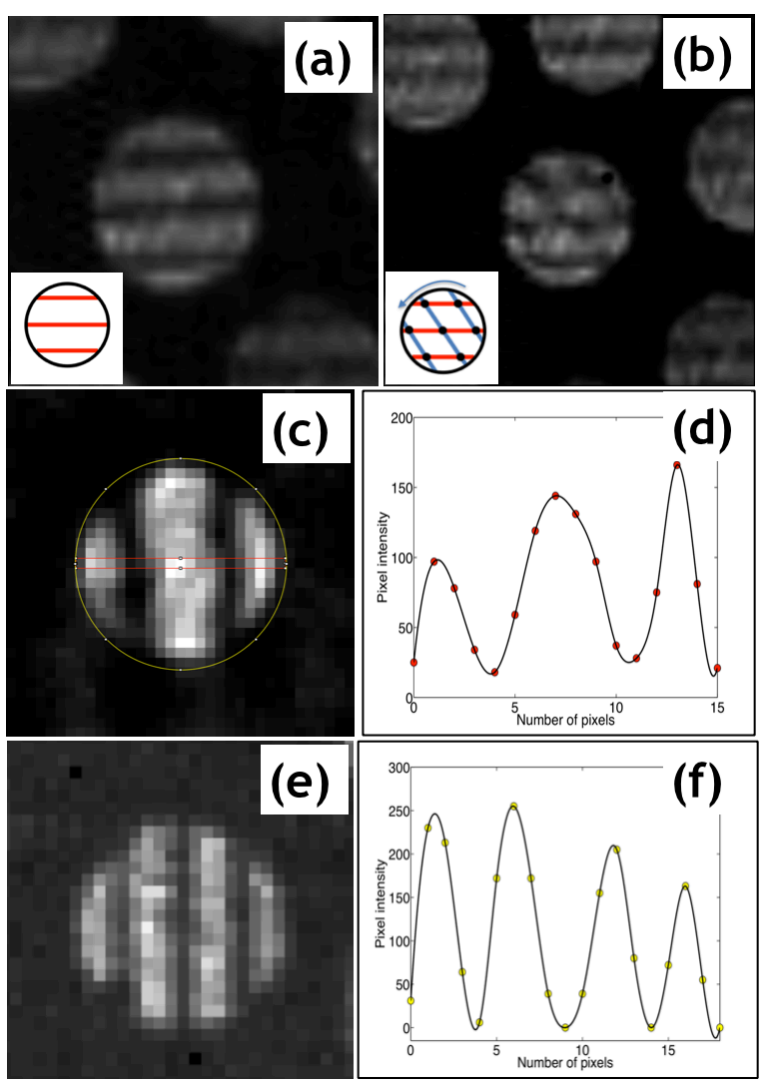

FIG. 4. (a) Overlap of rotational and stationary fringe patterns for a binary agglomerates. It shows that inter-fringe spacings are same for both types of rings patterns. (b) Nonoverlap fringe orientation for binary agglomerates where rotational fringes are oblique w.r.t stationary horizontal fringe patterns. The intersection of these two types of fringes form local dark patches on defocused images as illustrated in the inset by the black dots. (c) Illustration to calculate number of fringes for $11.35 \mu \mathrm{m}$ particle. In this case we consider rotational fringe patterns with region of interest across the diameter and perpendicular the fringe orientation. Intensity variation along the ROI has been observed where black pixels correspond fringe position. Fringe separation is determined as the distance between two maxima (or minima) of the intensity distribution. The number of fringes has been obtained by dividing the diameter of the particle (length of ROI) with fringe separation. (d) Intensity variation for $11.35 \mu \mathrm{m}$ diameter particle. Similar calculations have been performed for the bigger $20 \mu \mathrm{m}$ diameter particles where stationary fringes are formed as shown in (e) and (f). The fringe positions have been flipped to make clear visual effects. It is to be noted that the rotational interference fringe shape for binary agglomerate is not "exactly" vertical as observed in stationary fringes for spherical particles which can be due to morphological effect.

176 ference between the reflected and refracted rays can be 177 expressed as [50, 51]:

$$
\phi_{0}-\phi_{1}=\frac{2 \pi d}{\lambda}\left(\sin \frac{\theta}{2}-\sqrt{m^{2}+1-2 m \cos \frac{\theta}{2}}\right)
$$

178 An infinitesimal variation of the scattering angle induces 


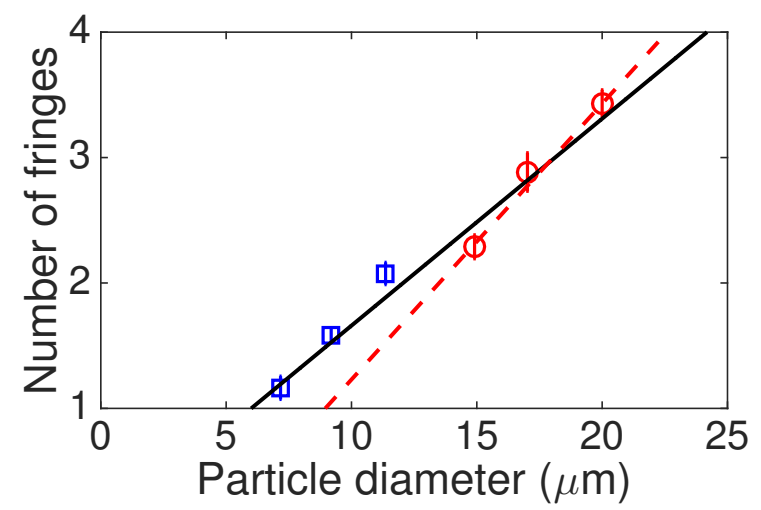

FIG. 5. Number of fringes $(\mathrm{N})$ on defocused images of particles increases with particle diameter $(d)$. Red circles represent the measurements using stationary fringe patterns on spherical particles. The red dash line represents linear fit with the data and provides a lower limit $d_{c} \sim 9 \mu \mathrm{m}$ below which no stationary fringe pattern on spherical particle is observable. Blue squares represent the measurement using fringe pattern on binary agglomerates. The solid line represents the best linear fit using all data points combining defocused image analysis of spherical particles and binary agglomerates, $\mathrm{N}=$ $0.17 d+0.01$, which provides an estimate of critical diameter of particle $d_{c} \sim 5.8 \mu \mathrm{m}$ below which no rotational fringe pattern on binary agglomerates is observable.

179 a maximum or minimum light intensity variation when 180 the infinitesimal phase difference is equal to $2 \pi$. So, the 181 angular inter-fringe spacing $\Delta \theta$ can be related to the par182 ticle diameter:

$$
\Delta \theta=\frac{2 \lambda}{d}\left(\cos \frac{\theta}{2}+\frac{m \sin \frac{\theta}{2}}{\sqrt{m^{2}+1-2 m \cos \frac{\theta}{2}}}\right)^{-1}
$$

183 If the scattering angle is of $90^{\circ}$ then it can be assumed 184 that the incidence angle of the refracted ray on the par185 ticle is close to zero and hence the above equation can be 186 simplified as,

$$
\Delta \theta=\left(\frac{2 \lambda}{d}\right) \frac{1}{1+\frac{1}{m}}
$$

${ }_{187}$ The number of fringes on the defocused image of a spher${ }_{188}$ ical particle depends on the collection angle, $\alpha$ which is ${ }_{189}$ equal to the product of number of fringes $N$ and angular 190 fringe spacing, $\Delta \theta$ :

$$
d=\left(\frac{2 \lambda N}{\alpha}\right) \frac{1}{1+\frac{1}{m}}
$$

191 The results have been described in Table-1 in which the ${ }_{192}$ left most column represents the diameter of the particles 193 as specified by the manufacturer. Then Eqn. 4 has been 194 used to estimate the particle diameters in experiments for 195 known refractive indices of materials, wavelength of the ${ }_{196}$ illuminating lasers and specified diameters (14.91, 17.02 197 and $20 \mu \mathrm{m})$.
198 It is to be noted that the diameters for smaller par199 ticles $(7.16,9.19$ and $11.35 \mu \mathrm{m})$ have been estimated by 200 analyzing rotational interference fringes on defocused im201 ages of their binary agglomerates. It is based on the 202 conjecture that the same measurement technique as de${ }_{203}$ scribed above for spherical particles is also applicable for 204 defocused image analysis of binary agglomerates which 205 contain rotational fringe patterns. This is due to the 206 fact that the inter-fringe spacing for rotational pattern 207 and stationary patterns are same for bigger size particles 208 where both patterns are visible. This simplified approxi209 mation agrees well with experimental observations. How210 ever, the full understanding of the source of these rotat${ }^{211}$ ing fringes is still unknown and kept for future work, but 212 they certainly represent the dynamic signatures of binary 213 agglomerates. It is found that the estimated diameters 214 are sufficiently close to those of specified diameters with ${ }^{215}$ maximum tolerance of $\sim 14 \%$ for $11.35 \mu \mathrm{m}$ particles and 216 minimum of $\sim 2 \%$ for $14.91 \mu \mathrm{m}$ particles.

\begin{tabular}{|c|c|c|c|c|c|}
\hline $\begin{array}{c}\mathrm{d}(\mu \mathrm{m}) \\
\text { Specified }\end{array}$ & Material & $\begin{array}{c}\mathrm{RI} \\
(\mathrm{m})\end{array}$ & $\begin{array}{c}\text { Defocused } \\
\text { image } \\
\text { analysis }\end{array}$ & $\begin{array}{c}\mathrm{N}: \text { \# of } \\
\text { fringes }\end{array}$ & $\begin{array}{c}\mathrm{d}(\mu \mathrm{m}) \\
\text { Estimated }\end{array}$ \\
\hline 7.16 & MF & 1.68 & BA & $1.16 \pm 0.09$ & $7.43 \pm 0.64$ \\
\hline 9.19 & MF & 1.68 & BA & $1.58 \pm 0.05$ & $10.12 \pm 0.32$ \\
\hline 11.35 & PS & 1.58 & BA & $2.07 \pm 0.09$ & $12.90 \pm 0.50$ \\
\hline 14.91 & MF & 1.68 & SP, BA & $2.28 \pm 0.09$ & $14.60 \pm 0.52$ \\
\hline 17.02 & PMMA & 1.49 & SP, BA & $2.88 \pm 0.15$ & $17.57 \pm 0.89$ \\
\hline 20 & PMMA & 1.49 & SP, BA & $3.43 \pm 0.10$ & $20.91 \pm 0.61$ \\
\hline
\end{tabular}

TABLE I. Particle diameter has been estimated using Eqn.4 by counting the number of interference fringes $(\mathrm{N})$ on defocused images of different spherical particles (SP) or binary agglomerates (BA) of different refractive indices $(\mathrm{m})$ but with same laser wavelength $(\lambda \sim 660 \mathrm{~nm})$ and collection angle $\left(\alpha \sim 23^{\circ}\right)$

${ }_{217}$ In conclusion, we have discussed a simple and useful 218 method to estimate size of a spherical particle over a 219 wide size range by analyzing defocused images of both 220 spherical particles and their binary agglomerates. The 221 diameter of the spherical particle has been estimated for 222 the first time by counting the number of interference ${ }_{223}$ fringes and their separation in the framework of inter224 ferometric laser imaging methods. The stationary fringe ${ }_{225}$ pattern is distinct for bigger spherical particles but they 226 are not clearly visible for medium size particles. To 227 overcome this problem, the separation of the rotating 228 fringes for the binary agglomerates has been used in 229 this size range. The number of fringes increases with 230 particle size and there exists two critical diameters below 231 which we do not observe any stationary and rotational 232 fringe patterns. This simple technique can be used to 233 identify size and shape of impurities or polydispersities 234 in laboratory experiments as well as under microgravity 235 conditions. 
* Email: manischaudhuri@g.harvard.edu
${ }_{238}$ Acknowledgement: M.C is supported by Marie-Curie 239 international outgoing fellowship.
271 [17] H. M. Thomas and G. E. Morfill, Nature 379, 806 (1996).

272 [18] V. E. Fortov, A. V. Ivlev, S. A. Khrapak, A. G. Khrapak, 329 273 and G. E. Morfill, Phys. Rep. 421, 1 (2005).

274 [19] S. V. Vladimirov and K. Ostrikov, Phys. Rep. 393, 175 $275 \quad(2004)$.

276 [20] U. Konopka, G. E. Morfill, and L. Ratke, Phys. Rev. 277 Lett. 84, 891 (2000).

278 [21] M. Lampe, R. Goswami, Z. Sternovsky, S. Robertson,

[22] M. Chaudhuri, S. A. Khrapak, and G. E. Morfill, Phys. Plasmas 14, 022102 (2007).

23] M. Chaudhuri, S. A. Khrapak, and G. E. Morfill, Phys. 340 Plasmas 15, 053703 (2008).

24] M. Chaudhuri, S. A. Khrapak, R. Kompaneets, and G. E. Morfill, IEEE Trans. on Plasma Sci. (2010). 343

25] G. E. Morfill, A. V. Ivlev, and H. M. Thomas, Phys. 344 Plasmas 19, 055402 (2012).

[26] R. A. Quinn and J. Goree, Phys. Rev. E 64, 051404 (2001).

27] M. Zuzic, A. V. Ivlev, J. Goree, G. E. Morfill, H. M. Thomas, H. Rothermel, U. Konopka, R. Sütterlin, and 34 D. D. Goldbeck, Phys. Rev. Lett. 85, 4064 (2000).

350 (2004).
P. M. Chaikin and T. Lubensky, Principles of Con- 297 densed Matter Physics (Cambridge University Press, 298 299
300 $301[31]$ 302 $304 \quad 99,025002$ (2007).

305 [33] C. A. Knapek, D. Samsonov, S. Zhdanov, U. Konopka, ${ }_{306}$ and G. E. Morfill, Phys. Rev. Lett. 98, 015004 (2007).

307 [34] S. Nunomura, D. Samsonov, S. Zhdanov, and G. Morfill, $308 \quad$ Phys. Rev. Lett. 95, 025003 (2005).

[35] B. Liu and J. Goree, Phys. Rev. Lett. 100, 055003 (2008). 310 [36] W.-T. Juan, M.-H. Chen, and L. I, Phys. Rev. E 64, 016402 (2001).

2 [37] G. E. Morfill, H. M. Thomas, U. Konopka, H. Rothermel, M. Zuzic, A. Ivlev, and J. Goree, Phys. Rev. Lett. 83, 1598 (1999).

[38] M. Kretschmer, S. A. Khrapak, S. K. Zhdanov, H. M. Thomas, G. E. Morfill, V. E. Fortov, A. M. Lipaev, V. I. Molotkov, A. I. Ivanov, and M. V. Turin, Phys. Rev. E 71, 056401 (2005).

[39] M. Rubin-Zuzic, G. E. Morfill, A. V. Ivlev, R. Pompl, B. A. Klumov, W. Bunk, H. M. Thomas, H. Rothermel, O. Havnes, and A. Fouquet, Nat. Phys. 2, 181185 (2006).

[40] H. M. Thomas, G. E. Morfill, V. E. Fortov, A. V. Ivlev, V. I. Molotkov, A. M. Lipaev, T. Hagl, H. Rothermel, S. A. Khrapak, R. K. Suetterlin, M. Rubin-Zuzic, O. F. Petrov, V. I. Tokarev, and S. K. Krikalev, New Journal of Physics 10, 033036 (2008).

[41] M. Schwabe, K. Jiang, S. Zhdanov, T. Hagl, P. Huber, A. V. Ivlev, A. M. Lipaev, V. I. Molotkov, V. N. Naumkin, K. R. Stterlin, H. M. Thomas, V. E. Fortov, G. E. Morfill, A. Skvortsov, and S. Volkov, Europhysics Letters 96, 55001 (2011).

[42] E. Thomas, Phys. Plasmas 6, 2672 (1999).

33 [43] E. Thomas and J. Williams, Phys. Plasmas 13, 055702 (2006).

[44] M. Kroll, S. Harms, D. Block, and A. Piel, Phys. Plasmas 15, 063703 (2008).

37 [45] B. M. Annaratone, T. Antonova, D. D. Goldbeck, H. M. Thomas, and G. E. Morfill, Plasma Physics and Controlled Fusion 46, B495 (2004).

[46] S. Kading and A. Melzer, Physics of Plasmas 13, 090701 (2006).

42 [47] M. Chaudhuri, V. Nosenko, C. Knapek, U. Konopka, 43 A. V. Ivlev, H. M. Thomas, and G. E. Morfill, Applied Physics Letters 100, 264101 (2012).

345 [48] M. Maeda, T. Kawaguchi, and K. Hishida, Meas. Sci. Technol. 11, L13 (2000).

34 [49] H. V. de Hulst, Light scattering by small particles (Wiley, 1957).

[50] C. Mounaïm-Rousselle and O. Pajot, Optical Technology in Fluid, Thermal and Combustion III 3172, 700 (1997).

351 [51] M. Golombok, V. Morin, and C. Mounaïm-Rousselle, J. Phys. D: Applied Physics 31, pL59 (1998). 